\title{
Access to Highly Tough Hydrogels by Polymer Modules for Application of Catalytic Reactors
}

\author{
Xiaofeng Niu, ${ }^{a}$ Yu Wang, ${ }^{a}$ Chengyuan Xu, ${ }^{b}$ Zhinan Fu, ${ }^{a}$ Shengyu Bai, ${ }^{a}$ Jie Wang, ${ }^{a}$ \\ Yiming Wang, ${ }^{a *}$ and Xuhong Guo ${ }^{a, c *}$
}

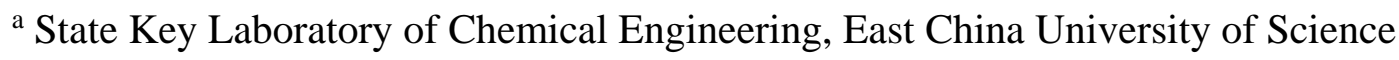 and Technology, 200237 Shanghai, P.R. China

${ }^{\mathrm{b}}$ Shanghai Key Laboratory of Chemical Biology, School of Pharmacy, East China University of Science and Technology, 200237 Shanghai, P.R. China

${ }^{\mathrm{c}}$ Engineering Research Center of Materials Chemical Engineering of Xinjiang Bingtuan, Shihezi University, 832000 Xinjiang, P.R. China

\footnotetext{
${ }^{*}$ To whom correspondence should be addressed. E-mail:

yimingwang@ecust.edu.cn (Yiming Wang); guoxuhong@ecust.edu.cn (Xuhong Guo)
} 
Table S1. The melting temperature, crystallinity and melting enthalpy calculated from DSC curves of PVA/PAA-T hydrogels prepared at different annealing temperatures. All hydrogels are fabricated with PAA content of $1 \mathrm{wt} \%$ and annealing for $1 \mathrm{~h}$.

\begin{tabular}{cccc}
\hline $\begin{array}{c}\text { Annealing } \\
\text { temperature } /{ }^{\circ} \mathrm{C}\end{array}$ & $\mathrm{T} m{ }^{\mathrm{a} /} \mathrm{C}$ & $\Delta H /\left(\mathrm{J} \mathrm{g}^{-1}\right)$ & Crystallinity (\%) \\
\hline 30 & 216.20 & 30.25 & 21.83 \\
100 & 216.15 & 38.78 & 27.99 \\
110 & 214.72 & 40.91 & 29.00 \\
120 & 217.20 & 45.62 & 32.91 \\
130 & 216.44 & 52.68 & 38.01 \\
140 & 217.33 & 51.87 & 37.42 \\
150 & 215.41 & 54.32 & 39.19 \\
\hline
\end{tabular}




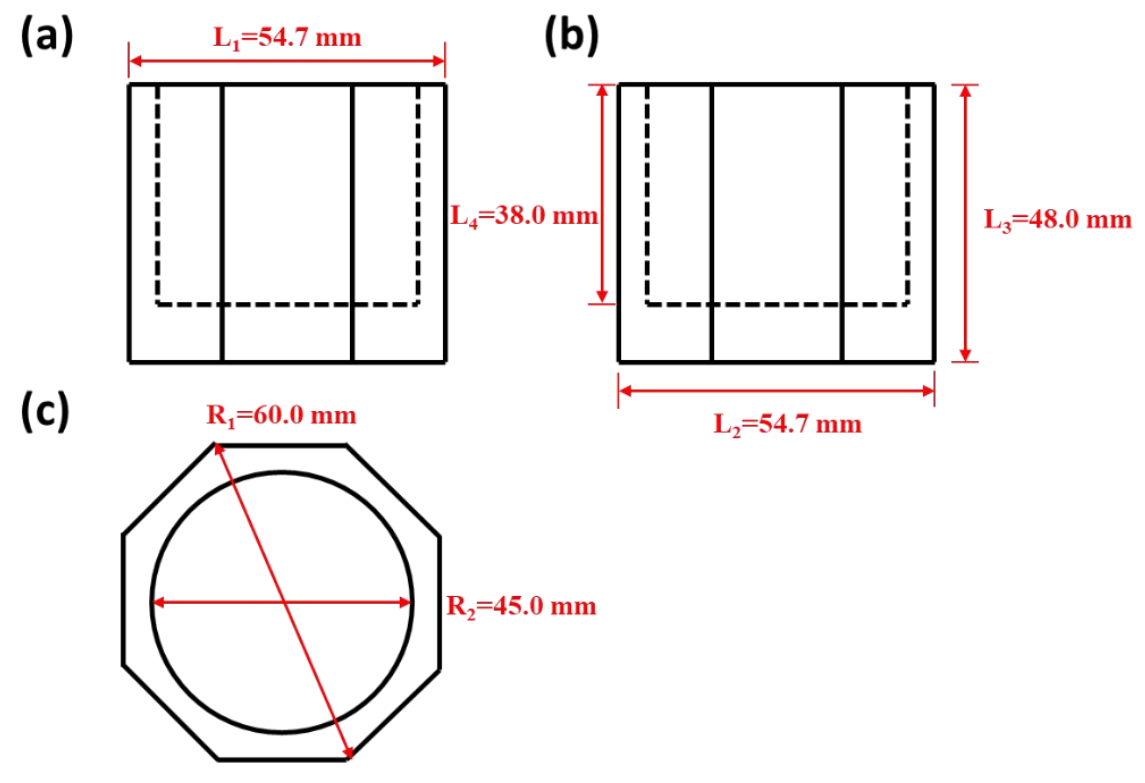

(d)
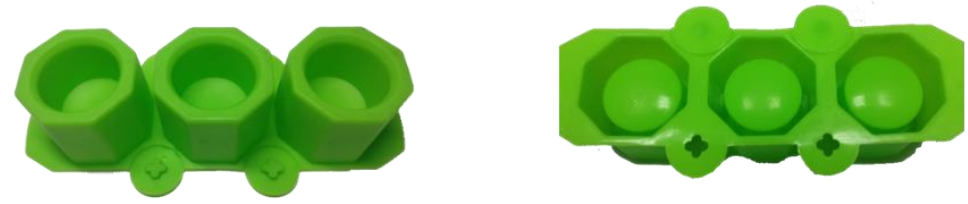

Figure S1. Three view drawing of designed Fenton-reactor. (a) Front view; (b) lateral view; (c) top view;(d) photographs of reactor mold. 
(a)

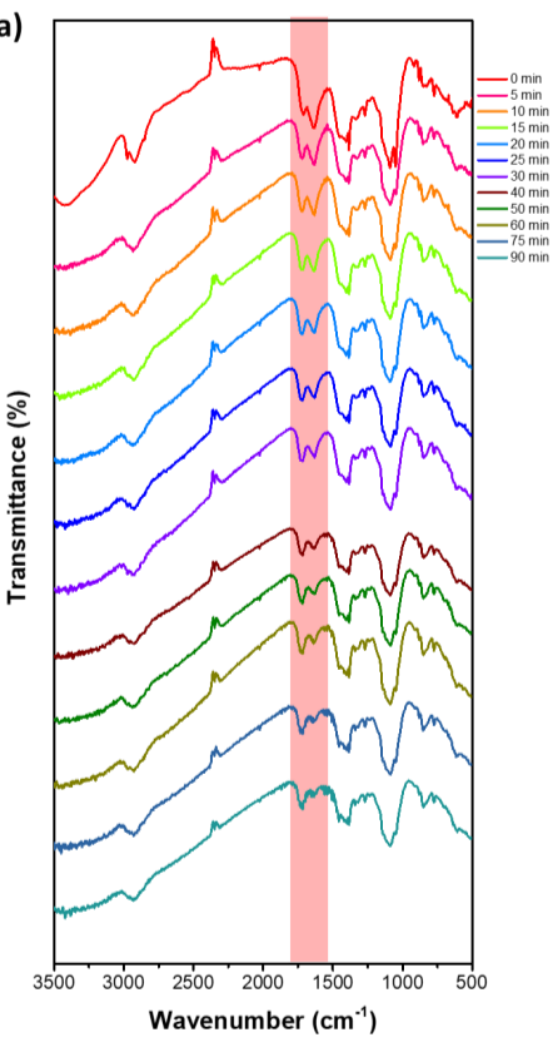

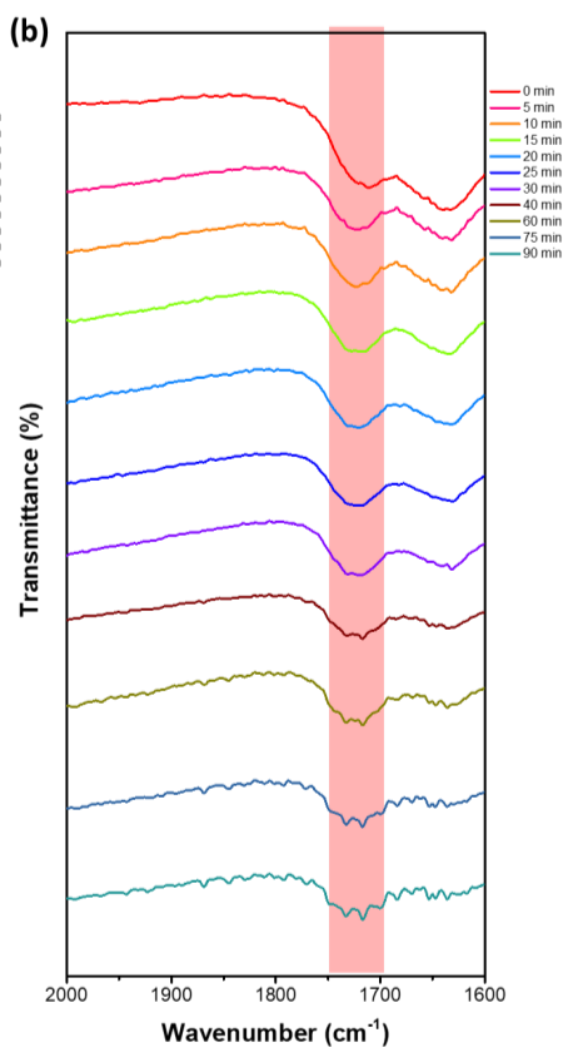

Figure S2. (a) Time-dependent FT-IR spectra of the PVA/PAA hydrogels with PAA content of 1 wt $\%$ under annealing temperature $\left(130{ }^{\circ} \mathrm{C}\right)$ in the time range of 0 - $90 \mathrm{~min}$; (b) enlarged view from 1600 to $2000 \mathrm{~cm}^{-1}$ of (a). 
(a)

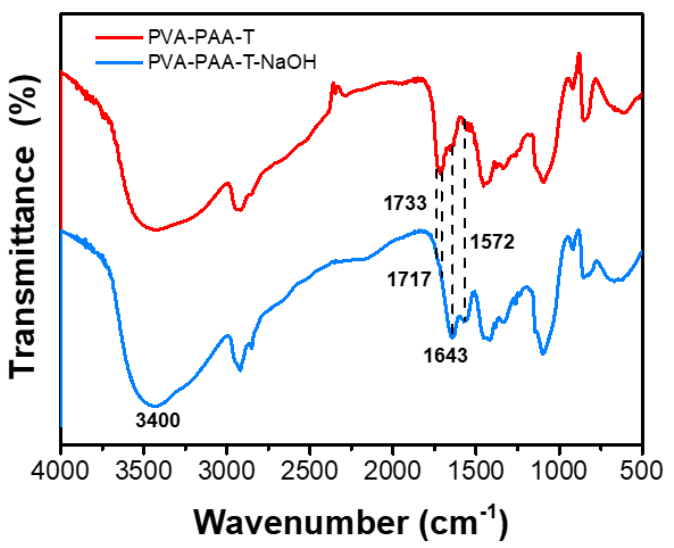

(b)

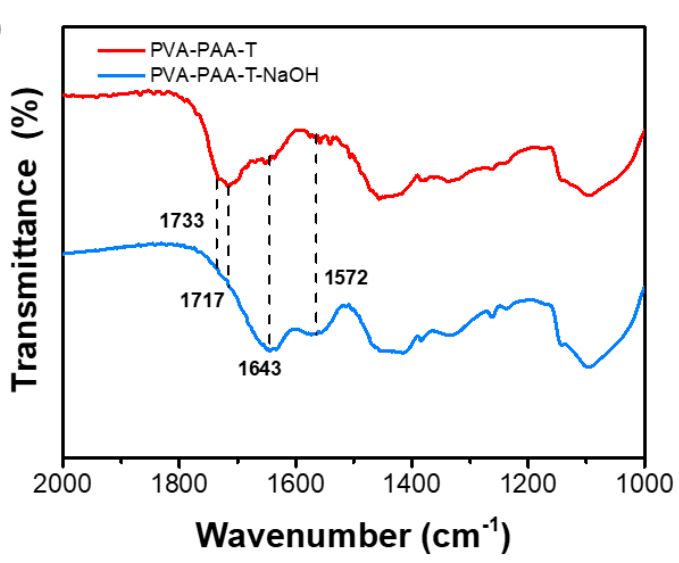

Figure S3. (a) FT-IR spectra of the PVA/PAA-T hydrogels and the PVA/PAA-T-NaOH hydrogels (the PVA/PAA-T hydrogel treated with $1 \mathrm{M} \mathrm{NaOH}$ solution for 7 days); (b) enlarged view from 1600 to $2000 \mathrm{~cm}^{-1}$ of (a). 


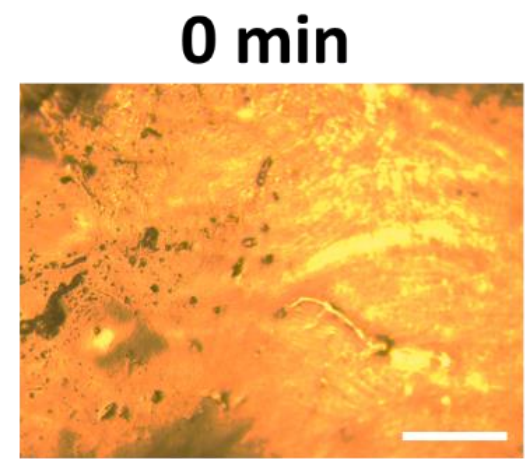

\section{Return to RT}

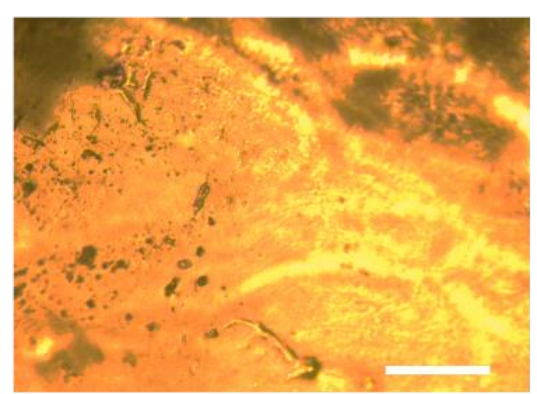

Figure S4. Optical microscope of PVA/PAA-T hydrogels before and after annealing at $130^{\circ} \mathrm{C}$ for 120 min. 
(a)
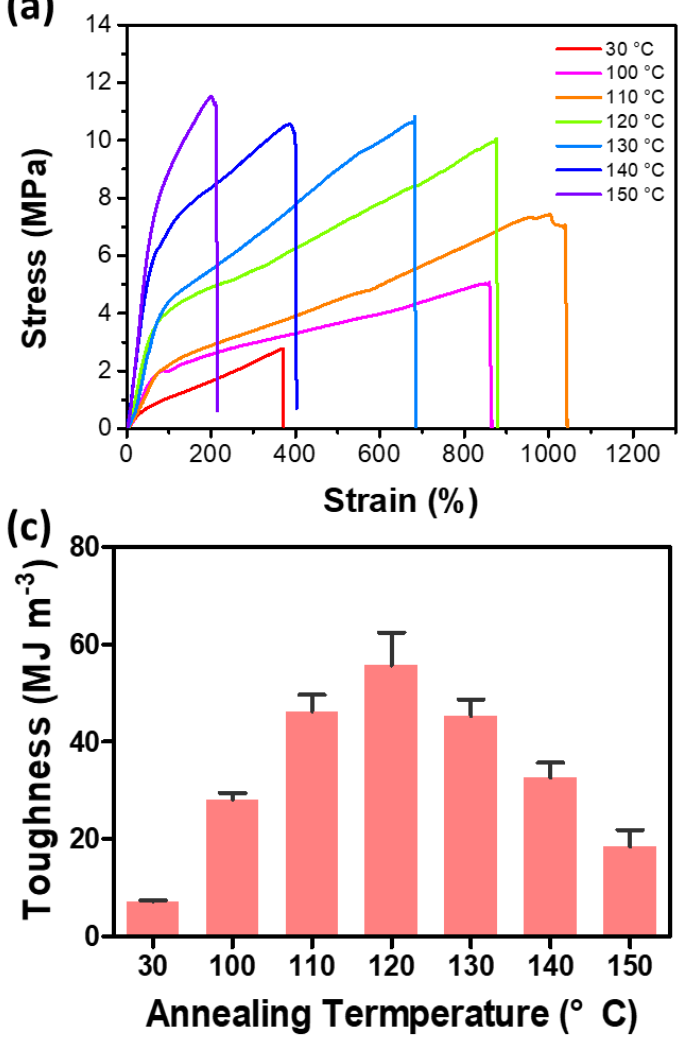

(b)

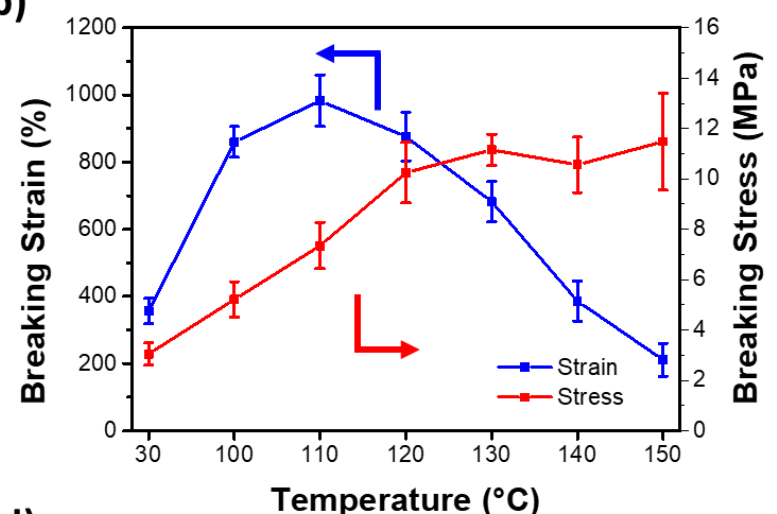

(d)

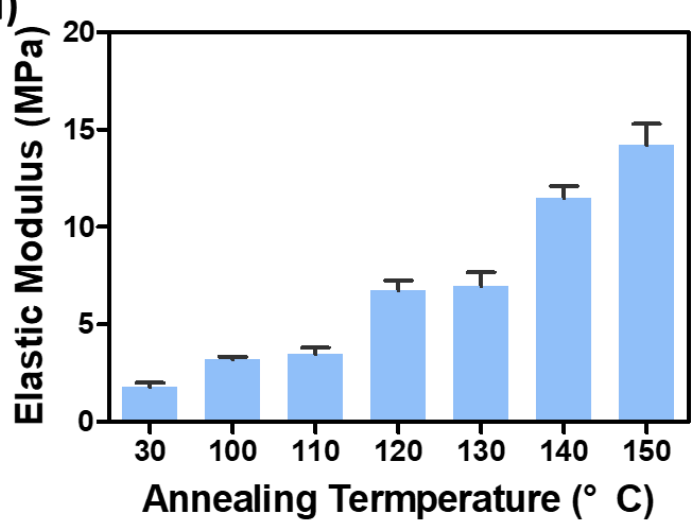

Figure S5. (a)The tensile stress-strain curves of PVA/PAA-T- $\mathrm{Fe}^{3+}$ hydrogels prepared at different annealing temperatures; (b) breaking strains and breaking stress changes over annealing temperatures; (c) toughness and (d) elastic modulus of PVA/PAA-T-Fe ${ }^{3+}$ hydrogels as function of annealing temperatures. All hydrogels are fabricated with PAA content of $1 \mathrm{wt} \%$ at and treated with $0.1 \mathrm{M} \mathrm{Fe}^{3+}$ solution. 


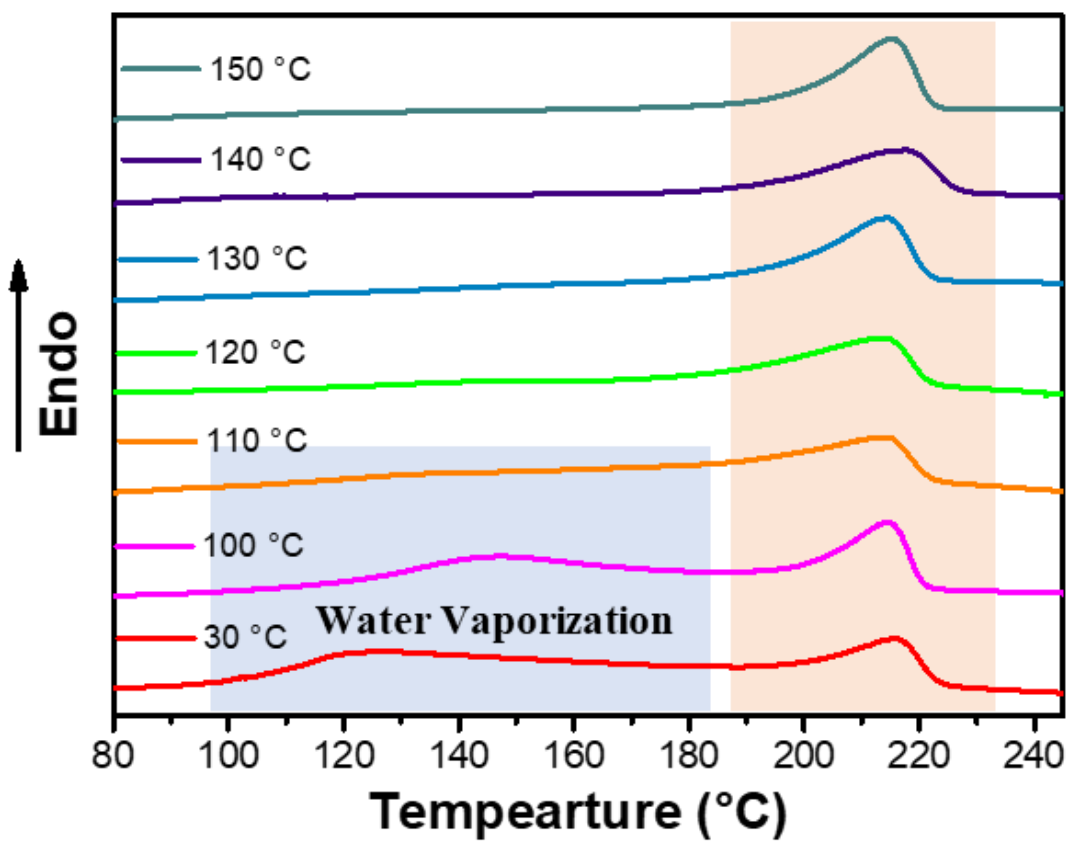

Figure S6. DSC Curves of PVA/PAA-T hydrogels prepared with different annealing temperatures.

All hydrogels with PAA content of $1 \mathrm{wt} \%$ are treated with annealing at for 1 hour. 

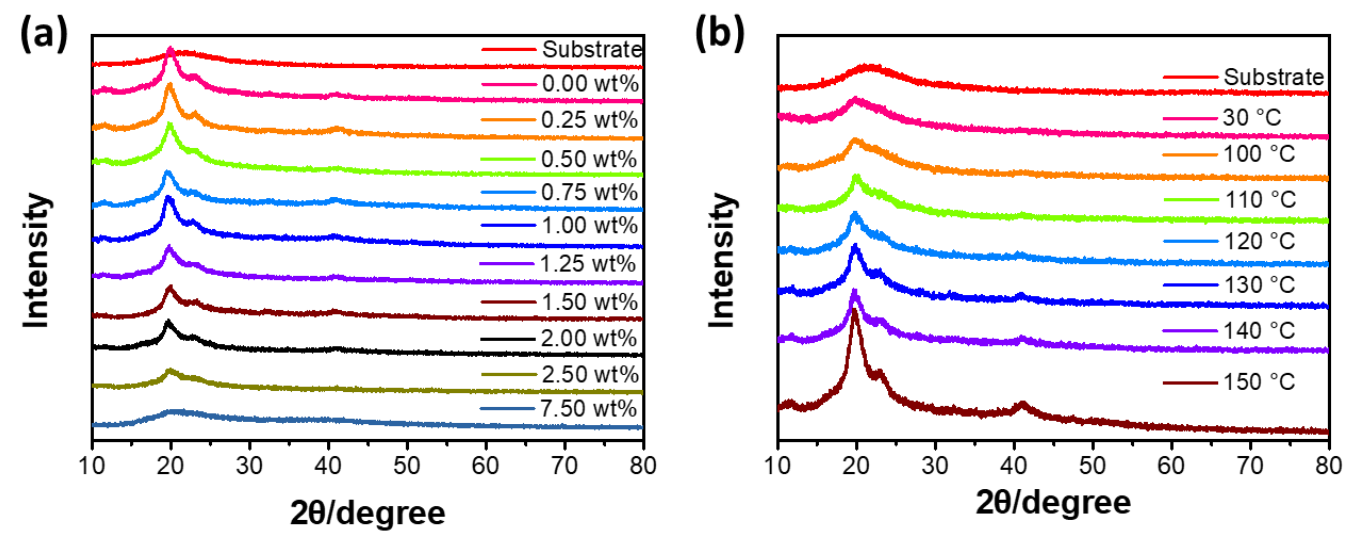

Figure S7. (a) X-ray diffraction profiles of the PVA/PAA-T hydrogel prepared with different PAA contents; All hydrogels are treated with annealing at $130{ }^{\circ} \mathrm{C}$ for 1 hour; (b) X-ray diffraction profiles of the dried PVA/PAA-T hydrogel with different annealing temperatures for 1 hour. All hydrogels are fabricated with PAA content of $1 \mathrm{wt} \%$. 

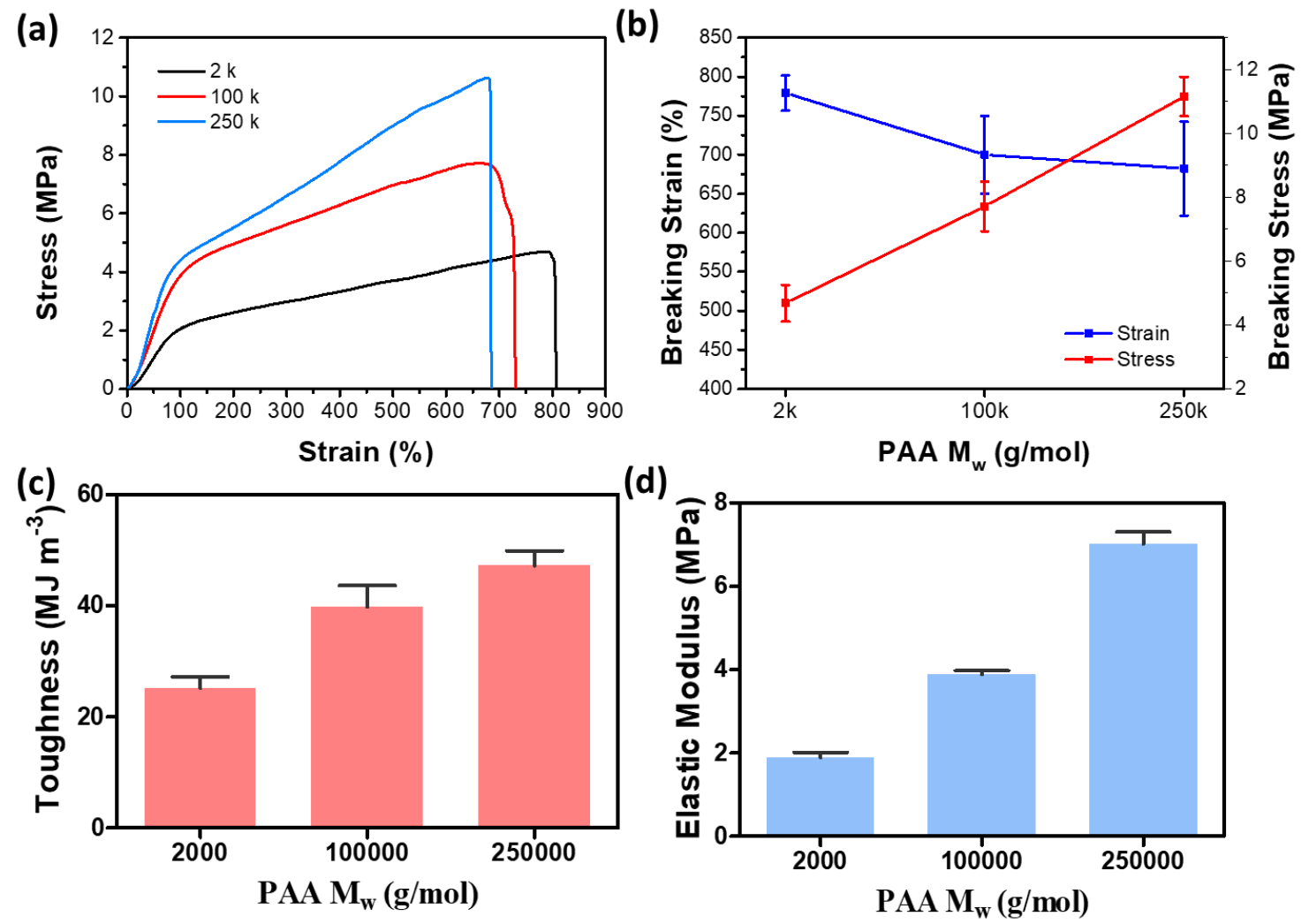

Figure S8. (a)The tensile stress-strain curves of PVA/PAA-T-Fe ${ }^{3+}$ hydrogels prepared with different molecular weights of PAA; (b) breaking strains and breaking stress changes over molecular weights of PAA; (c)toughness and (d) elastic modulus of PVA/PAA-T- $-\mathrm{Fe}^{3}$ hydrogels as function of molecular weights of PAA. All hydrogels are treated with annealing at $130^{\circ} \mathrm{C}$ for 1 hour and $0.1 \mathrm{M} \mathrm{Fe}^{3+}$. 
(a)
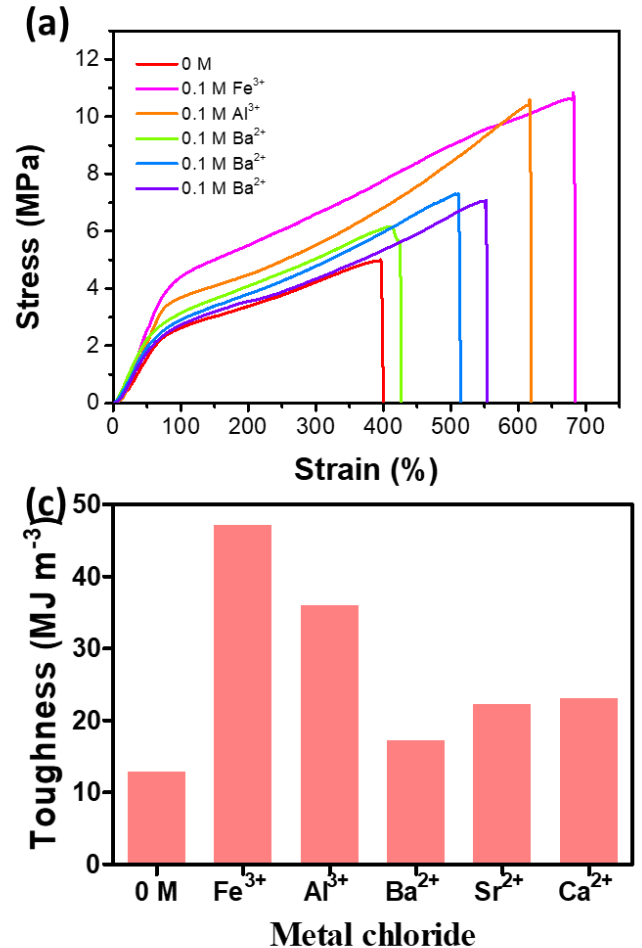

(b)
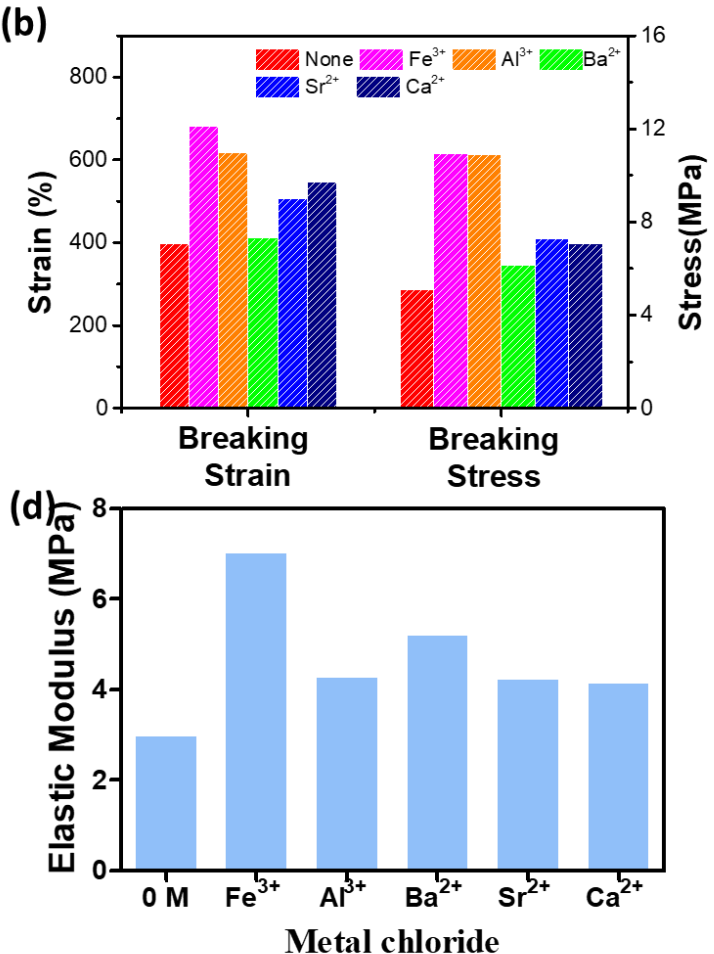

Figure S9. (a)The tensile stress-strain curves of PVA/PAA-T-M ${ }^{\mathrm{n}+}$ hydrogels prepared with different types of metal cation; (b) breaking strains and breaking stress changes over types of metal cation; (c)toughness and (d) elastic modulus of PVA/PAA-T-M ${ }^{\mathrm{nt}}$ hydrogels as function of types of metal cation. All hydrogels are fabricated with PAA content of $1 \mathrm{wt} \%$, annealing at $130{ }^{\circ} \mathrm{C}$ for 1 hour and treated with $0.1 \mathrm{M} \mathrm{M}^{\mathrm{n}+}$ solution. 


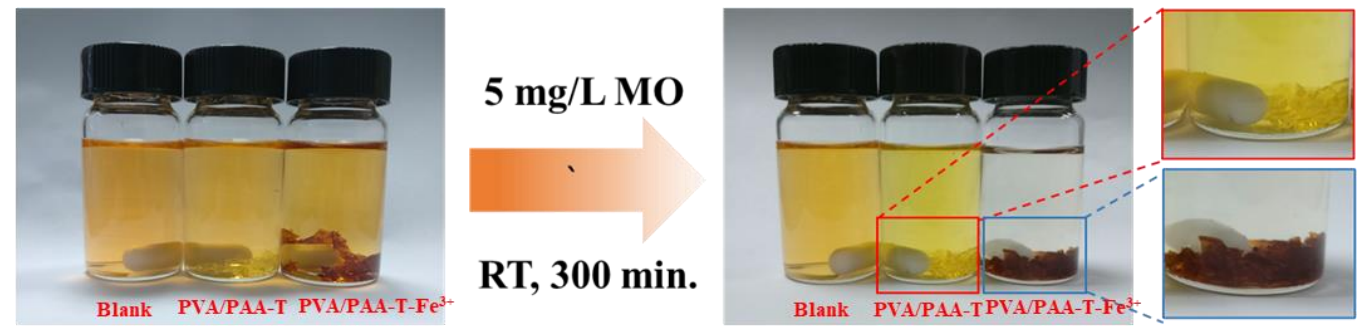

Figure S10. Photographs of Fenton reaction over different samples (Blank, PVA/PAA-T hydrogels, PVA/PAA-T-Fe ${ }^{3+}$ hydrogels). 

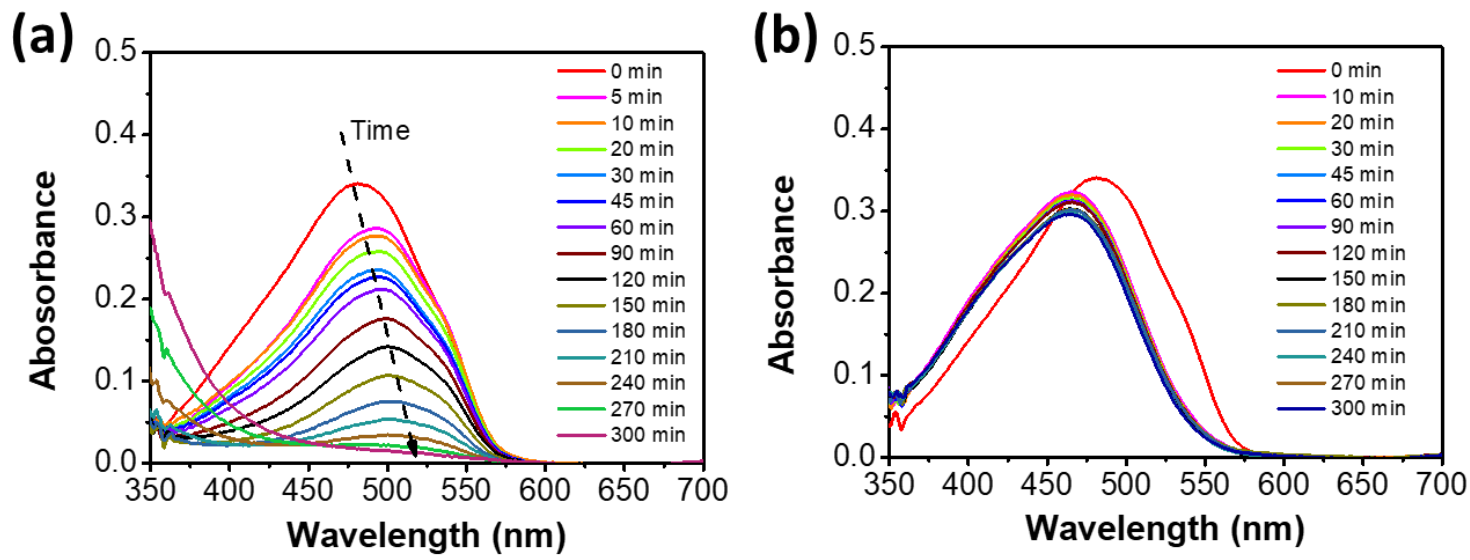

Figure S11. UV-vis absorption spectra of the dye solutions incubated in the reactors made by (b) PVA/PAA-T-Fe ${ }^{3+}$ hydrogels and (c) PVA/PAA hydrogels; (d) degradation of MO in different containers. 
<smiles>CN(C)c1ccc2nc3ccc(=[N+](C)C)cc-3sc2c1</smiles>

Methylene Blue (MB)

Figure S12. Molecular structures of methylene blue (MB).

(a)

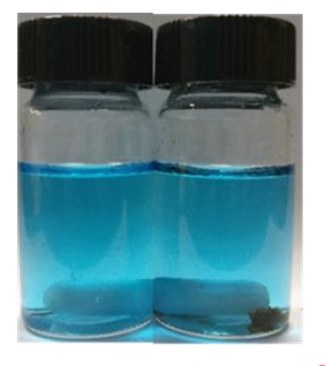

$12 \mathrm{~h}, \mathrm{RT}$

(b)

Blank PVA/PAA-T-Fe ${ }^{3+}$

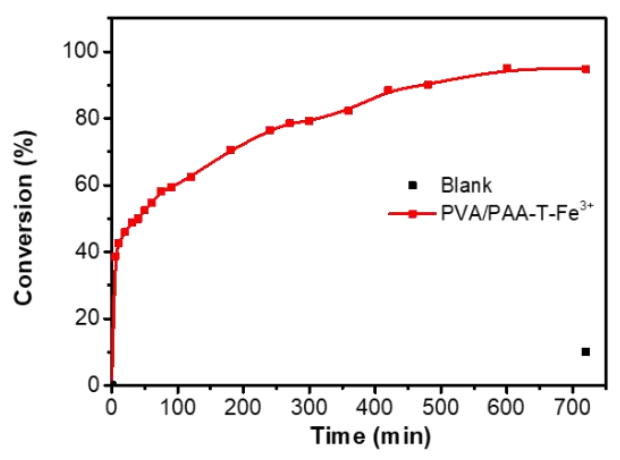

(c)

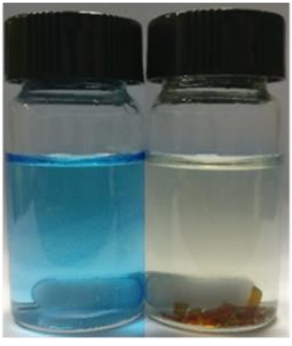

Blank PVA/PAA-T-Fe ${ }^{3+}$

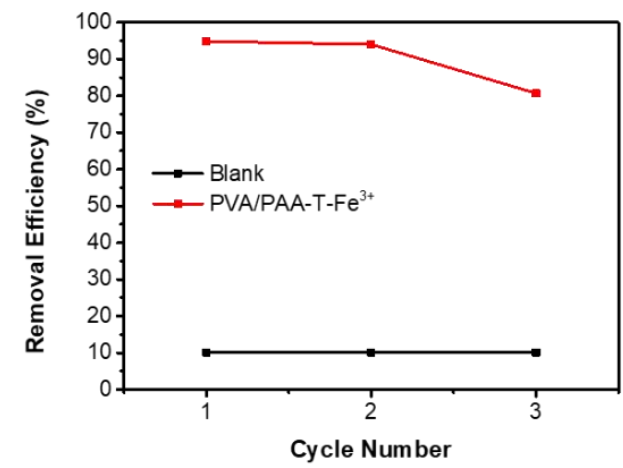

Figure S13. (a) Photograph of Fenton reaction performed by PVA/PAA-T-Fe ${ }^{3+}$ hydrogels; (b) the catalytic degradation of MB under catalyzed by hydrogels over time; (c) renewability of the hydrogels for the catalytic degradation of MB. All Fenton reactions: $5 \mathrm{mg} / \mathrm{L} \mathrm{MB}$, initial $\mathrm{pH}$ of $5.96,0.60 \mathrm{~g}$ of hydrogels and room temperature. The control experiment is carried out in the absence of hydrogels. 